\title{
Sumo Robot Wheeled based Remote Control using Telecommunications Module the Frequency of $433 \mathrm{MHz}$
}

\author{
${ }^{1}$ Indah Trizkiyani, ${ }^{1}$ Tito Waluyo Purboyo, ${ }^{2}$ Simon Siregar and ${ }^{2}$ Muhammad Ikhsan Sani \\ ${ }^{1}$ Department of Computer Engineering, Faculty of Electrical Engineering, \\ ${ }^{2}$ Department of Computer, Faculty Applied Science, Telkom University, Bandung, Indonesia \\ indahrz18@gmail.com
}

\begin{abstract}
Robot is a set of mechanical devices that could do physical tasks like human and mostly applied as research. One of them with capability of motion like human beings is the sumo robot. Sumo robot is the type robot vehicles taking analogy competition martial arts sumo from Japan. There are two categories of sumo robot that are the autonomous and RC (Remote Control) and categories the heavy robots there are three categories that are 1,3 and $5 \mathrm{~kg}$. The pilot should have expertise in control remote control. This study discussed sumo robot category with heavy $3 \mathrm{~kg}$ and custom communication system. It's takes a communication system of sumo qualitative robot by remote control. Qualitative system is making of protocol data that has specific function and has a frequency equal to data transmission handshaking model. Qualitative system can use the model TCP/IP OSI because is one to one that data transmission will not have mixed up or called handshaking model. A kind of remote control used that is a play station 2 joystick and module of communication that is used namely APC220 module and regulated by way of the frequency of $433 \mathrm{MHz}$. In addition, it can display the capacity of voltage sumo robot with an indicator LED who has 10 bars, buzzer and uses voltage sensor and current sensor ACS712.
\end{abstract}

Key words: Sumo robot, joystick play station 2, APC220 module, current sensor ACS712, voltage sensor, TCP/IP modul

\section{INTRODUCTION}

Robot there has been many involved in the lives of daily securely, good robot with supervision control of man or automatically. One of them with capability of motion like human beings is the sumo robot (Ciamoscki et al., 2015). The sumo robot divided into two categories autonomous and RC (Remote Control). Sumo robot also have to push or drop their opponent off the arena when robot managed to shove or drop their opponent out of the ring, so, the robot expressed win.

The sumo robot category RC (Remote Control), need strategy and expertise of a human being or commonly called pilot in play. Therefore, sumo robot controlled by a pilot should has communication system that qualitative by remote control. A communication system qualitative namely by make a protocol of the data have specific function each other that is by using model protocol data TCP/IP and use the submission of the data with a model handshaking and has a frequency equal robot sumo by remote control. If not having equal frequencies a robot not be able to communicate $w$ ith remote control (Lima et al., 2013). Therefore, needed module communication who works at the frequency of $433 \mathrm{MHz}$. Module communication that can be worked at the frequency of $433 \mathrm{MHz}$ one of them radio module APC220 (Siregar et al., 2017).

With the techniques or methods used it is very important that in order to calculate, assess, classify, etc. on a digital filter will result in an effective and efficient design. All electronic filters work by applying digital mathematical operations or algorithms to a signal processing. One of the main limits on digital filters is in terms of the limitation of processing speed/computational time that is highly dependent on the capabilities of the microcontroller or the computer used.

Basic theory: Handshaking protocol is the process of communication between two different devices before normal communication begins (Victor et al., 2013). Its

Corresponding Author: Indah Trizkiyani, Department of Computer Engineering, Faculty of Electrical Engineering,

Telkom University, Bandung, Indonesia, indahrz18@gmail.com 


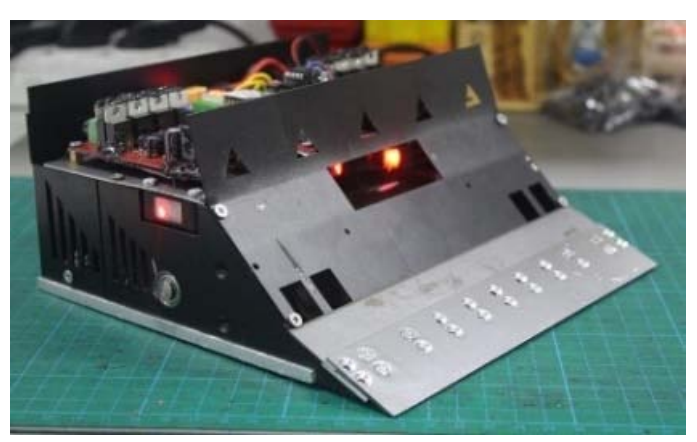

Fig. 1: Sumo robot model (Orefice et al., 2016)

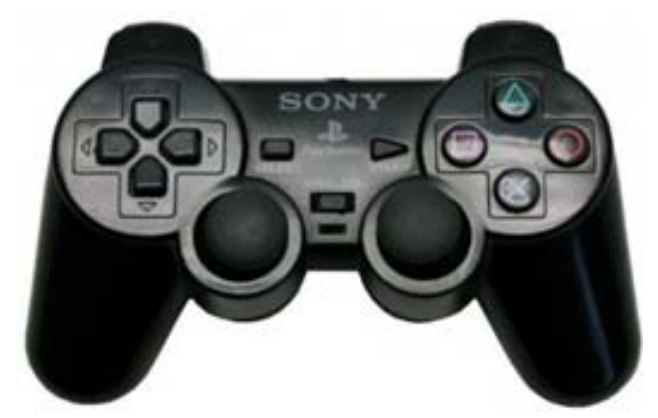

Fig. 2: Play station 2 joystick (Al-Jarrah and Abdel-Karim, 2006)

functions are to exchange information protocols and to prove the quality of connections and authorities needed to complete the connection between devices. The handshaking protocol is used in the sumo robot system which is for communication between the remote-control system and sumo robot system.

Sumo robot: Sumo robot is the robot sport in which two robots pushing each other out of a circle (in a similar to sports sumo). Robots used in this competition called sumobots (Fig. 1).

Joystick play station 2: The joystick is a remote to control the robot and has a custom input button, so, the robot can move in all directions. A joystick consisting of some switches as X button, $\square$ button, $\Delta$ button, o button, left right up down button, L1, L2, R2, R1, SELECT, START, X and $\mathrm{Y}$ axis button (Fig. 2). The Play Station 2 (PS2) joystick is very friendly and the PS2 joystick remote control unit can be played easily as a pilot. Therefore, 21 function joysticks native mapped into 8 digital and buttons analog joystick 2. Because there is a reduced mapping maybe this way, the number of functions it was chosen and only draw for research programmed into software (Erdem, 2011).

The cable to configuration on the PS2 joystick there are 9 cables that have their own colors. For the first brown cable that functions as data sent or input on the joystick button. The second cable is orange which functions to receive data from the sumo robot. The third gray cable serves as power on the robot motor that receives an output of around 7-9 V. Then the fourth cable is black as ground and the fifth cable is red as a power or VCC that has a size of $3.3 \mathrm{~V}$. Sixth cable is yellow as attention but not used in sumo robot. For the seventh and eighth cables not used it is usually blue as a clock and white. For the last cable the ninth serves as an ACK (Fig. 3).

Voltage sensor and sensor current ACS712: Voltage sensor is a gauge the current DC voltage. This module having maximum input voltage until $0-25 \mathrm{~V}$. Maximum voltage $25 \mathrm{~V}$ if desired input Arduino analogous to voltage $5 \mathrm{~V}$ and if to voltage $3,3 \mathrm{~V}$, voltage input must not more than 16, $5 \mathrm{~V}$. Having 3 pin the data, VCC and GND (Su et al., 2017). Current sensor ACS712 has 5A having hall-effect. Hall-effect designed to feel absence of object magnetic with the changes its position. ACS712 can measure current $\mathrm{AC}$ and $\mathrm{DC}$. The sensor is having a range sensitivity $0,066 \mathrm{~V} / \mathrm{A}$ up to $0,185 \mathrm{~V} / \mathrm{A} 3$. ACS7 12 has three pins namely VCC, GND and data (Fig. 4).

APC220 radio module: APC220 is module wireless telecommunications operator namely to versatile with a capacity of low which it is easy to be integrated with for disaster for any of the project who are in need of link radio frequency. This module suitable for this application is reasonable enough robotic add ons that could put into the robot are at the provincial level requires wirelessly. This module can be have joined to microcontroller through interface TTL (Transistors Transistors Logic) then have been join PC with APC220 module other through converter TTL/USB 4. Frequency in the APC220 module worked between $420-450 \mathrm{MHz}$. For setting frequency, node ID, etc. can use application RF-Truly Magical (Fig. 5).

APC220 is semi-duplex module, that can be communicated with pointed to one point to the multi-point. On second mode, the user need to set a single host module while the other one was module client. Every module is only allowed to having one unique ID. An inherent characteristic of the communication of APC220 wireless ran into financial difficulties of the delay in the wireless data communication on the control of flux, control the mistakes and choices of its antenna. (Bohnert et al., 2018).

LED bar and buzzer: Light Emitting Diode or LED is a component electronics that can exude monochromatic light when it is given voltage. Constituting the family 


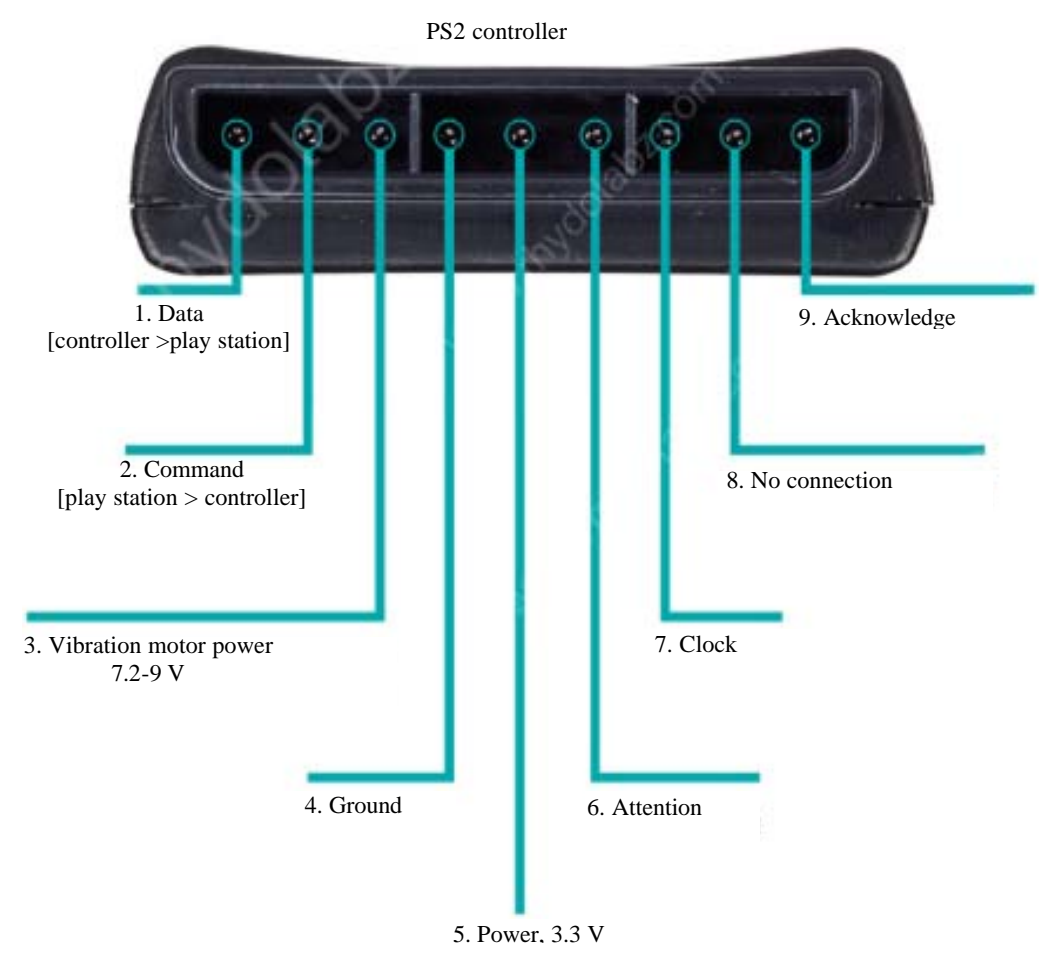

Fig. 3: Input-output cable PS2 joystick
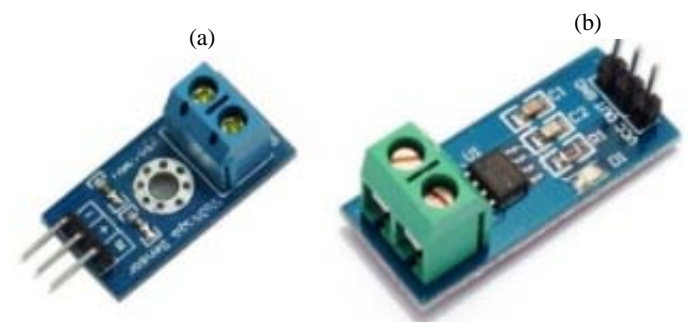

Fig. 4: a) Voltage sensor and b) Current sensor (Su et al., 2017)

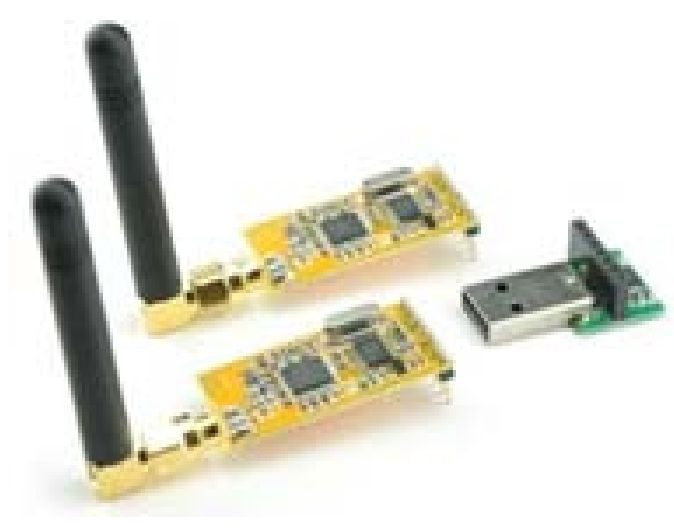

Fig. 5: APC220 module communication (Yamato et al., 2008)

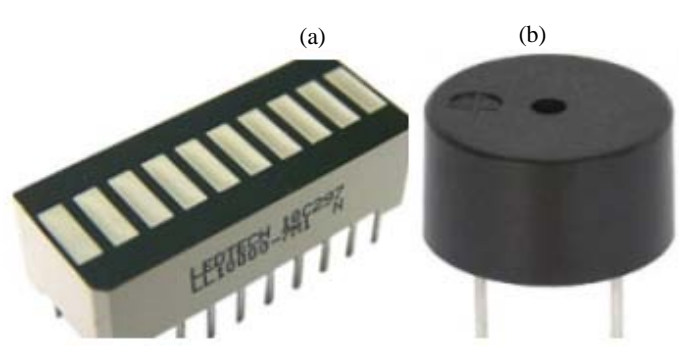

Fig. 6: a) LED bar and b) Buzzer (Bohnert et al., 2018)

LED, a diode made from semiconductor substance. LED bar been a set of the LED that same as led generally. But LED the shaped bar rectangular and lapped in a good shape. Buzzer is a component electronics that serves to change the vibration of electricity into vibrations of sound (Aydogan and Aras, 2017). Basically, the working principle of loud buzzer almost the same as the speaker. Buzzer has a voltage of input $5 \mathrm{~V}$ (Fig. 6).

\section{MATERIALS AND METHODS}

The image of the suggestions and design system: Framework of the design system was firstly, there is the system of play station input joystick when pressed a button will send data packets according to the Nano microcontroller Arduino present on remote control system 

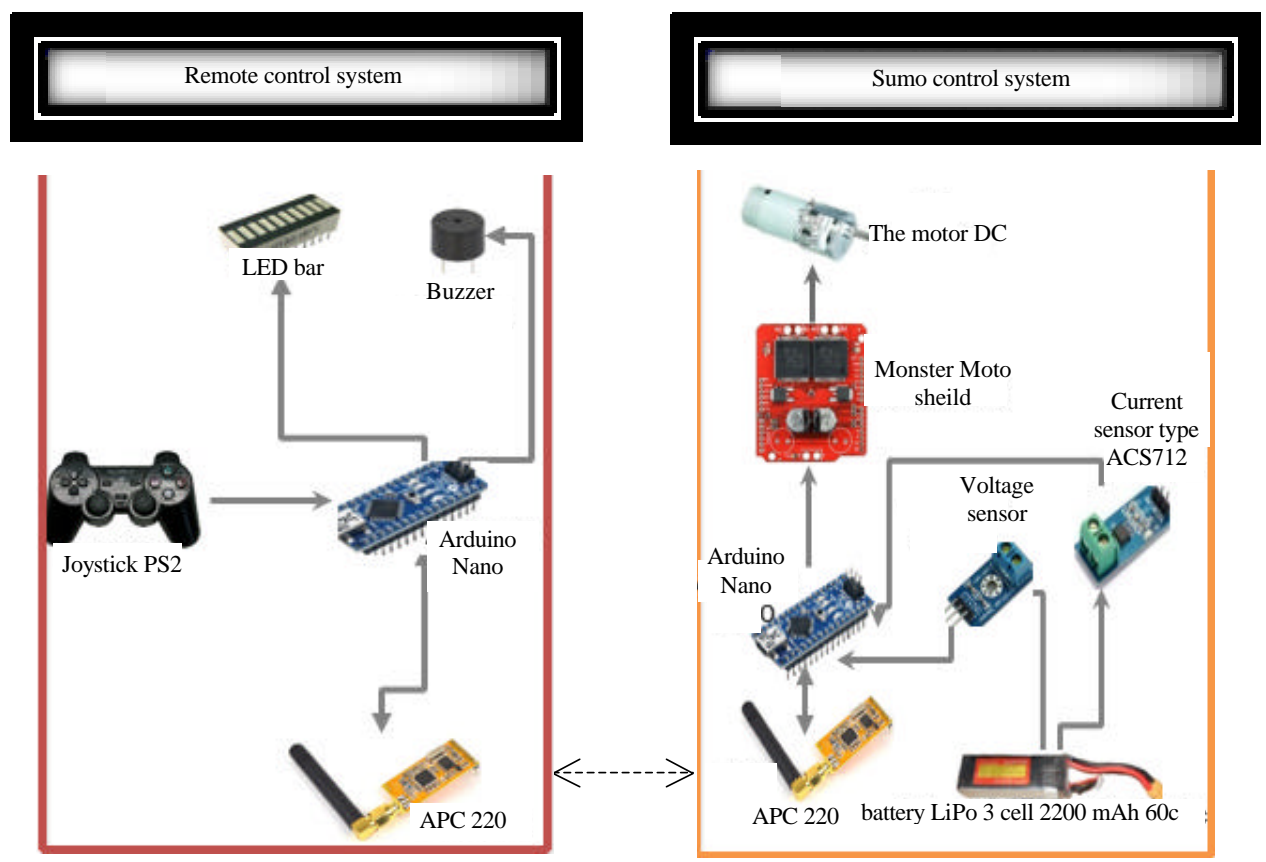

Fig. 7: The design sumo robot system

APC220 module communication and went through (Qidwai et al., 2013). Then, data packets that are received through communication on the robot APC220 module sumo will be processed to microcontroller which is with in robots after that, come into the driver motor monster's moto shield and run motor as ordered. After data packets fit in, sumo robot will send data packets of data value sensors voltage and the value of a current sensor data ACS712 through communication APC220 module. Data packets that are received through communication APC220 module on a remote-control system that passes through both microcontroller LED Bar and will be displayed in the buzzer. The first experiment is to measure the pressure that is given the movement at the time of shaking hands (Yamato et al., 2008) (Fig. 7).

The conclusion is the joystick sent data to the controller on arduino then the data was sent on a pair of APC 220 module. After that, it was received at the Arduino robot controller and the robot will run according to the instructions of the joystick. When the robot battery has reached $20 \%$, the current and voltage sensors will detect and then give commands and sent via. APC 220. when the command has arrived, the LED and buzzer will light up, so, the pilot knows if the battery is below $20 \%$.

TCP/IP (Transmission Control Protocol/Internet Protocol): The protocol is specification formal that defines procedures to be followed when transmit and receive data. TCP/IP having 4 layers to each other having protocol with function complementary to one another. Layers from above was making the process more interactive (Orefice et al., 2016). That means, TCP/IP consists of 4 layers in the form of a multilevel set of protocols that is a network access layer that is responsible for sending and receiving data from the interface. Internet layer that is responsible for sending to predefined addresses (IP, ARP and ICMP). The transport layer is responsible for communicating between hosts. and the last application layer is where applications that use the TCP/IP stack are located (Fig. 8).

Design a communication system: Figure 9 is visualizes sending data to the ACP on the joystick system to the sumo robot and vice versa. Model the submission of the data on either system use the model three-way handshake. The purpose of this method is to synchronize to deliver the ordinal number of transmitted data by either system. How it works can be described as follows:

Data transmission started with remote control or joystick PS2 to the robot as ordered of the pilot. First data packets PS2 joystick before being sent in encapsulation first, encapsulation is a way to hide detailed information data or packaging data after encapsulation data packets will be sent. If the system robots received data packets PS2 joystick, so, data packets will be decapsulation. 


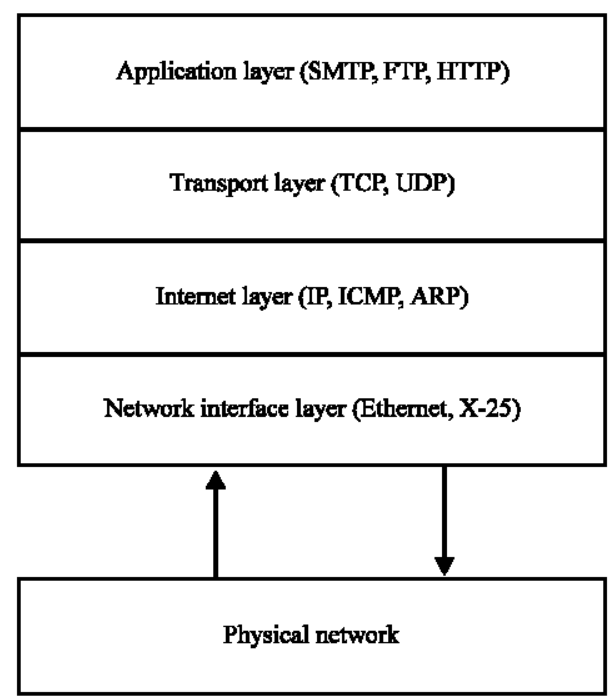

Fig. 8: Architecture TCP/IP (Gacharna et al., 2016)

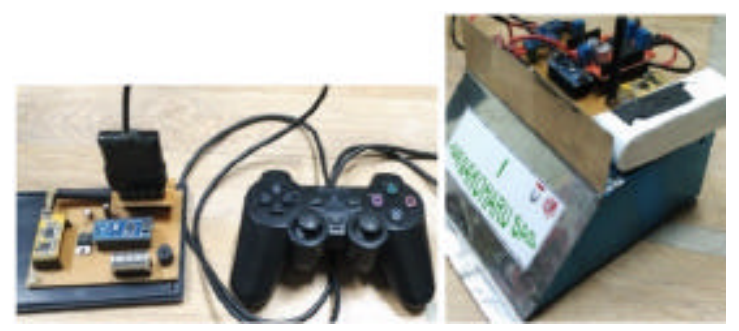

Fig. 9: Implementation Sumo robot system

Decapsulation is process of opening wrap data. If there are data beyond the cocoon of opened the contents of data packets. The contents of data packets called PDU was (Protocol Units) data that is going to be divided per unit of the data of every layer. After a PS2 joystick sent data, the robot will require sends data of censorship voltage and current sensors to the system remote control. Before, data packets robots sent first in encapsulation (packaging) data. If remote control system given to data will wrap the decapsulation (the opening data). Then displayed in indicators LED bar and buzzer. Delivery data are needed protocol data, model protocol data used is the model TCP/IP. Was broken module sets up stricter and interacting with their environment outside the system. Then the module as controlling and regulate security, provide all facilities needed to use as the achievement of a goal. Namely, the security of a third module data that is responsible to the contents of the data (Gacharna et al., 2016).

In layer transport break up data to packages data and give the ordinal number of every package, so that, may be prepared again after accepted. In layer this is happening the process encapsulation and decapsulation, a kind of the submission of the data layer transport is reliable. Reliable data is sending that allows checking error and a sending back. In layer transport give the ordinal number of on the data PS2 joystick and on the sensors voltage and current. Layer on the Internet work make header/marker on the packs amongst the data will be sent and checksum as markers. On layer was conducted detection error and transmission of repeated the packages are error. Mapping of the components that included in first and next namely control robots on USB-UIRT (Al-Jarrah and Abdel-Karim, 2006). Example; Header on a system remote control that is worth " $\mathrm{A}$ " and for system sumo robot is "B" (from ASCII) converted to say decimal, the checksum no worth of. On layer network serves put frame-frame data network will be sent to the media, one of the media tissue namely APC220 module.

\section{RESULTS AND DISCUSSION}

Installing and testing system: The installation of components and electronic module by using tin solder and wire. Components and putting the PCB module on board had already been printed in accordance to a pretty unvarying design has been designed. In a pairing should not be attaching solder too long on PCB board and components because electronic components can be damaging (Fig. 10).

To be able to do the submission of the data between the APC220 module then must by controlling the tissue on the device. To do the setting on device can use the application of RF-Truly Magical forest. It should be noted that net ID to be the same between device that will be totally interconnected here. Because net ID is the identity of a network of any device. All device that has net the same ID, so, it can communicate with. In addition, node ID must be different for each device because node ID is the identity of any device (Fig. 11).

First test that do is testing the wireless data systems in remote control to the sumo robot. Testing this, data transmission of PS2 joystick to robots to know whether the move as ordered already programmed in microcontroller. Following the outcome of the experiment of testing remote control (Table 1).

Pulse-Width Modulation (PWM) is a way of describing a digital (binary/discrete) signal. The results of the condition that is the robot will move wheels, moved wheels hanging from buttons PS2 joystick which is pressed. Meaning PWM had directed namely manipulate output digital in such a way, so, as to produce analogue signals. So that, moved robot if PWM the bigger, the robot will the sooner whereas if PWM the less moved robot will be more slowly. 
J. Eng. Applied Sci., 14 (18): 6587-6595, 2019

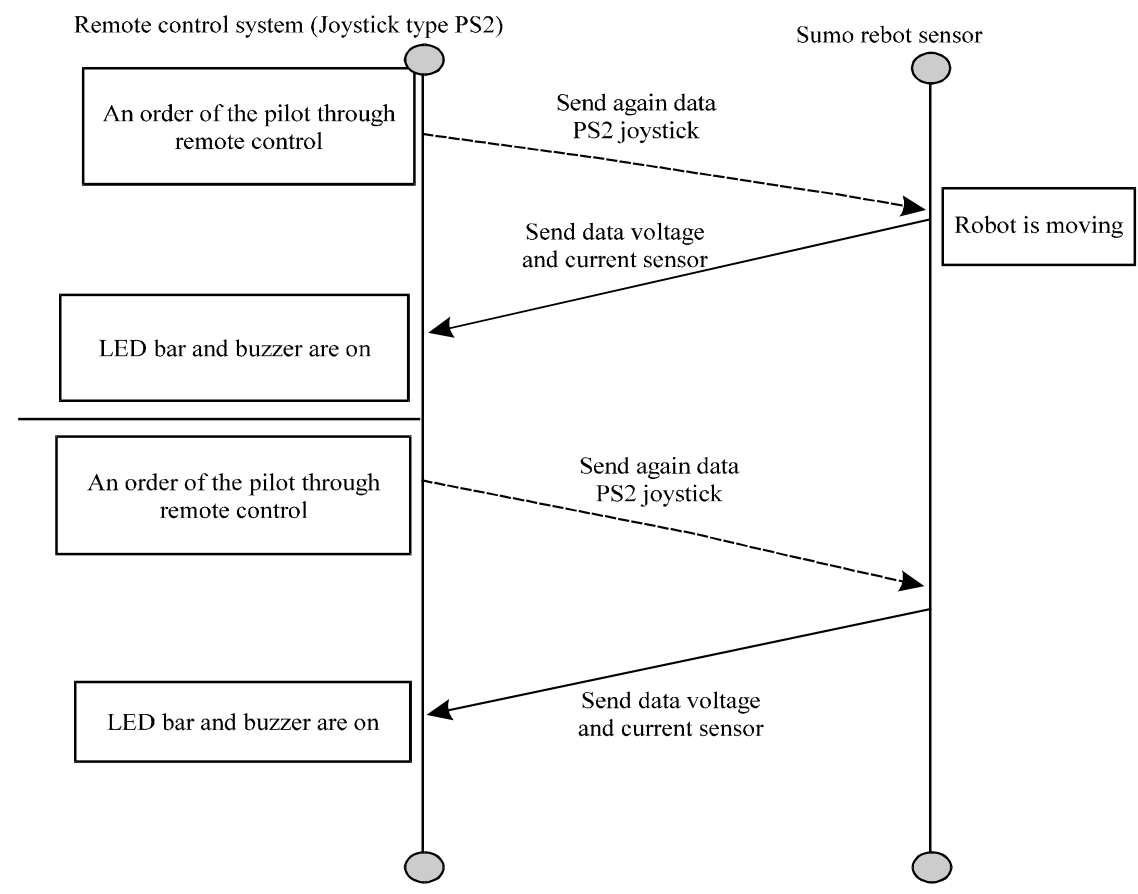

Fig. 10: Implementation sumo robot system

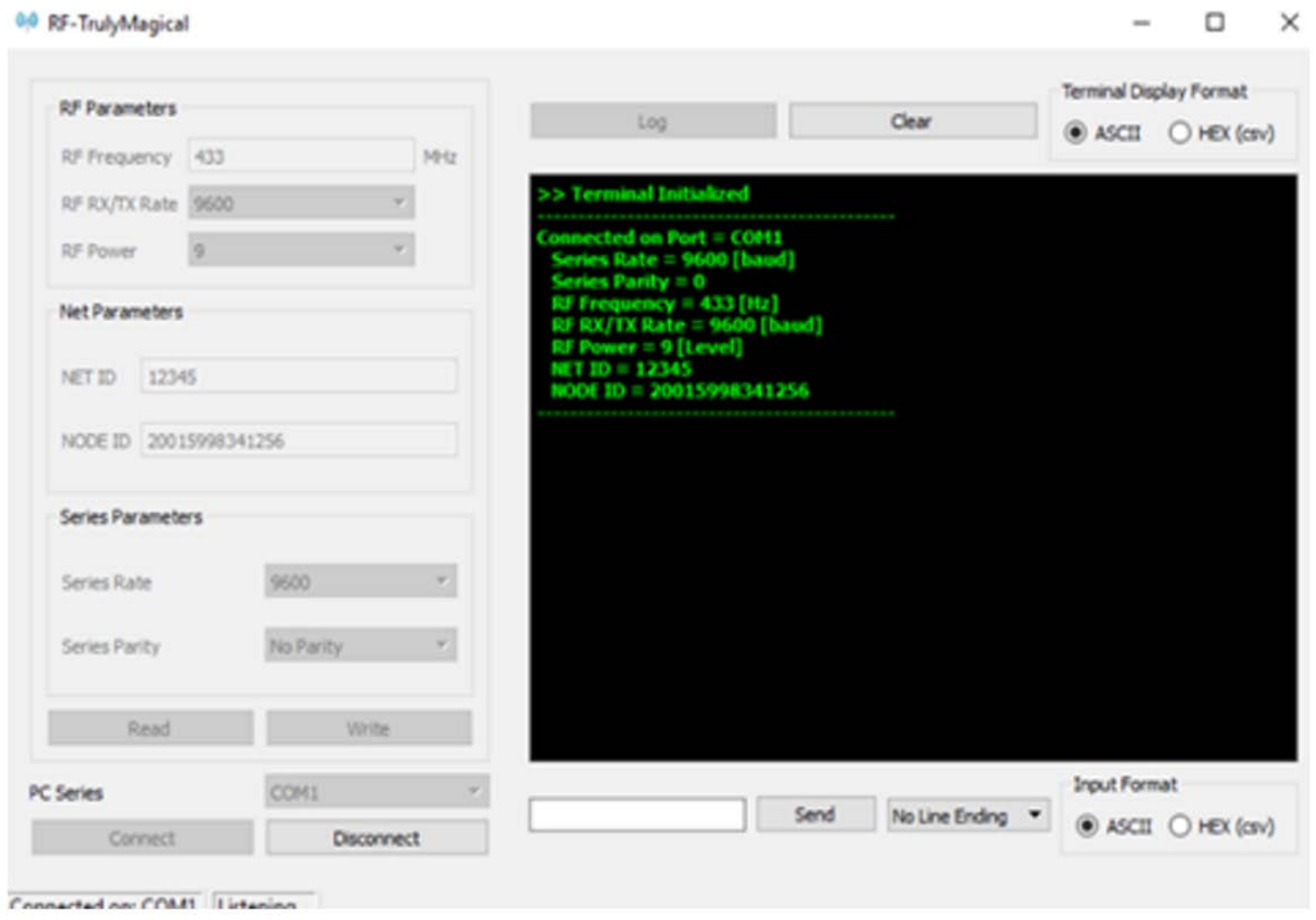

Fig. 11: Setting module APC220 use application RF-Truly Magical forest 


\begin{tabular}{|c|c|c|c|c|c|c|c|c|c|c|c|c|c|c|c|c|c|c|c|}
\hline \multirow{2}{*}{$\begin{array}{l}\text { Joystick button } \\
\text { Data }\end{array}$} & \multicolumn{19}{|c|}{ Joystick data sent to sumo robot } \\
\hline & 1 & 2 & 3 & 4 & 5 & 6 & 7 & 8 & 9 & 10 & 11 & 12 & 13 & 14 & 15 & 16 & 17 & Conditions results & Information \\
\hline UP & 0 & 0 & 0 & 0 & $\mathrm{FF}$ & 0 & 0 & 0 & 0 & 0 & 0 & 0 & 80 & 80 & 80 & 80 & 190 & Up with PWM: 220 & Work out \\
\hline Down & 0 & 0 & 0 & 0 & 0 & FF & 0 & 0 & 0 & 0 & 0 & 0 & 80 & 80 & 80 & 80 & 190 & Down with PWM:200 & Work out \\
\hline Left & 0 & 0 & 0 & 0 & 0 & 0 & FF & 0 & 0 & 0 & 0 & 0 & 80 & 80 & 80 & 80 & 190 & $\begin{array}{l}\text { Up and Left with } \\
\text { PWM: } 255\end{array}$ & Work out \\
\hline Right & 0 & 0 & 0 & 0 & 0 & 0 & 0 & $\mathrm{FF}$ & 0 & 0 & 0 & 0 & 80 & 80 & 80 & 80 & 190 & $\begin{array}{l}\text { Up and Right with } \\
\text { PWM: } 255\end{array}$ & Work out \\
\hline $\mathrm{X}$ & $\mathrm{FF}$ & 0 & 0 & 0 & 0 & 0 & 0 & 0 & 0 & 0 & 0 & 0 & 80 & 80 & 80 & 80 & 190 & $\begin{array}{l}\text { Down with PWM: } \\
220\end{array}$ & Work out \\
\hline 口 & 0 & 0 & $\mathrm{FF}$ & 0 & 0 & 0 & 0 & 0 & 0 & 0 & 0 & 0 & 80 & 80 & 80 & 80 & 190 & $\begin{array}{l}\text { Right and Down } \\
\text { With PWM: } 80\end{array}$ & Work out \\
\hline$\Delta$ & 0 & 0 & 0 & $\mathrm{FF}$ & 0 & 0 & 0 & 0 & 0 & 0 & 0 & 0 & 80 & 80 & 80 & 80 & 190 & $\begin{array}{l}\text { Left and Down } \\
\text { With PWM: } 80\end{array}$ & Work out \\
\hline$\circ$ & 0 & FF & 0 & 0 & 0 & 0 & 0 & 0 & 0 & 0 & 0 & 0 & 80 & 80 & 80 & 80 & 190 & $\begin{array}{l}\text { Left and Down } \\
\text { With PWM: } 80\end{array}$ & Work out \\
\hline L1 & 0 & 0 & 0 & 0 & 0 & 0 & 0 & 0 & 0 & 0 & $\mathrm{FF}$ & 0 & 80 & 80 & 80 & 80 & 190 & Up with PWM: 255 & Work out \\
\hline $\mathrm{L} 2$ & 0 & 0 & 0 & 0 & 0 & 0 & 0 & 0 & 0 & 0 & 0 & FF & 80 & 80 & 80 & 80 & 190 & Down with PWM:255 & Work out \\
\hline
\end{tabular}

The second testing: The sensor in data voltage wireless to the system remote control. Testing made by comparing data from its own power supply and with the results of the value of censorship voltage. Real data sensors voltage is the data on analogous thus the probability of faced with the condition input who flexible with a value continuous. Real data sensors voltage programmed using function map() real data of the value of 300-700 are the 4-12, 5 . Function map (the map input analog resolutions 300-700 to output analog resolution of 4-12, 5 volt. Value 4-12, 5 volts made to the equation of data power supply not far, data voltage power supply is comparison value voltage for measuring. The percentage capacity voltage battery obtained from a Eq. 1:

$$
\begin{aligned}
& \text { The percentage capacity voltage battery: } \\
& =\left|\frac{\text { Data voltage sensor-Capacity battery }}{\text { Capacity battery }}\right| \times 100 \%
\end{aligned}
$$

The meaning of produce values as the percentage of the recipe had battery voltage, the percentage is assessed absolute which means that it not be valued at negative. Then, calculation the percentage error or distinction the percentage error it means the difference in value error from real data voltage and the results of the function map() microcontroller program. The bigger the percentage error so module of sensors used not good or calibration made far from real data. The percentage error calculated from a Eq. 2 (Table 2):

The percentage error:
$=\left|\frac{\text { Data power supply-Data voltage (conversion) }}{\text { Data power sup ply }}\right| \times 100 \%$

The third testing: The submission of the data flow sensors ACS712 in wireless services to the system remote
Table 2: Testing the reception of the data the sensors that are in the manner of a voltage a wireless to the system a remote controller

\begin{tabular}{lccc} 
Measurement & Calculation & & \\
$\begin{array}{l}\text { Data from power } \\
\text { supply (v) }\end{array}$ & $\begin{array}{l}\text { The percentage } \\
\text { error voltage (\%) }\end{array}$ & $\begin{array}{c}\text { Data voltage } \\
\text { sensor (V) }\end{array}$ & $\begin{array}{c}\text { The percentage } \\
\text { capacity battery (\%) }\end{array}$ \\
\hline 4 & 17.25 & 4.69 & 37.52 \\
7 & 6.43 & 6.55 & 52.4 \\
9 & 13.11 & 7.82 & 62.56 \\
11 & 10.27 & 9.87 & 78.96 \\
12 & 4.80 & 11.90 & 95.2 \\
13 & 3.85 & 12.50 & 100 \\
\hline
\end{tabular}

control displayed serial monitor. Testing sensors flow was by changing data PWM on motor because the current influenced by burden. In its testing voltage input was set at $12 \mathrm{~V}$. The battery used as battery Lipo 3 cell (lithium polymer) having specification $2200 \mathrm{mAh}$ and $60^{\circ} \mathrm{C}$. The measurement result value some current does would be different far from the data before and after but (weight. The greater weight used the bigger currents and the less weight the less current. Value a current does affect the percentage capacity voltage battery on the robot (Table 3).

The fourth testing: The results reading led bar and buzzer. Calculation has been done to be seen on indicators led bar and buzzer. Scenario reading indicators led bar and buzzer namely originally input voltage battery (Battery Lipo) used namely 3 cell or a maximum of voltage until $\pm 12.5 \mathrm{~V}$. The result of voltage is to be divided to each bar that is in LED Bar. The following the results of testing of LED bar and buzzer. Based on the results of tests made in Table 4, at the time of battery fully loaded/full led bar will burn 10 bar and buzzer dead with the $100 \%$. While at the time of the percentage $95,2 \%$, LED bar will burn 9 bar and buzzer dead. The percentage $70,72 \%$ led bar on 6 bar and buzzer dead, if the percentage reached $<50 \%$ moved motor will slow. Buzzer would beep beep beep when conditions led bar on 3 bars 


$\begin{aligned} & \text { Table 3: Testing the submission of the data flow sensors ACS712 in } \\
& \text { wireless services to the system remote control displayed serial } \\
& \text { monitor }\end{aligned}$
$\begin{array}{llll}\text { PWM } \\
\text { (pulse width }\end{array}$
\begin{tabular}{llll} 
modulation) & Batterry 3 & Measurement & The result calculate \\
on robot & cell (V) & power supply (A) & Densor (A) \\
\hline 25 & 12 & 0.46 & 0.15 \\
50 & 12 & 0.80 & 0.40 \\
100 & 12 & 1.24 & 0.78 \\
150 & 12 & 1.30 & 1.05 \\
200 & 12 & 1.36 & 1.21 \\
255 & 12 & 1.44 & 0.90 \\
\hline
\end{tabular}

Table 4: Testing reading LED bar and buzzer

\begin{tabular}{llll}
\hline Voltage (V) & The percentage battery (\%) & LED bar & Buzzer \\
\hline $6.60-7.20$ & 4.8 & 2 bar & A long sound \\
$7.20-7.80$ & 46.32 & 3 bar & Bip Bip 3x \\
$7.80-8.40$ & 62.56 & 4 bar & Off \\
$9.00-9.60$ & 70.72 & 6 bar & Off \\
$10.80-11.40$ & 95.2 & 9 bar & Off \\
$>11.40$ & 100 & 10 bar & Off \\
RTO (Request & - & blink & Bip Bip 3x
\end{tabular}

Timed Out)

and that at $46,32 \%$ and buzzer rang tone long when conditions LED bar on 2 bars about $4.8 \%$ and if the data not sent or occurring RTO (Request Timed Out), buzzer will would beep beep beep and LED bar on blink.

\section{CONCLUSION}

From the results of the testing robot's sumo and the system of remote control so a conclusion can be drawn as follows: the data sent by the system remote control type 2 play station accepted by the sumo robot, so that, it can move motor/the robot by command received robot. Wireless of radio frequency $433 \mathrm{MHz}$ can communicate in both directions, so, called transceiver because it could serve as transmitter (sender) and receiver (recipients), so that, it can be monitoring uses the battery led bar and buzzer with sensors voltage and current ACS 712 sensors.

\section{REFERENCES}

Al-Jarrah, M. and R.T. Abdel-Karim, 2006. A thin security layer protocol over IP Protocol on TCP/IP suite for security enhancement. Proceedings of the International Conference on Innovations in Information Technology, November 19-21, 2006, IEEE, Dubai, United Arab Emirates, pp: 1-5.

Aydogan, H. and F. Aras, 2017. Design of a graphical user interface for analysis of a fiber optic current sensor. Proceedings of the 2017 10th International Conference on Electrical and Electronics Engineering (ELECO), November 30-December 2, 2017, IEEE, Bursa, Turkey, ISBN: 978-1-5386-1723-6, pp: 1332-1335.
Bohnert, K., C.P. Hsu, L. Yang, A. Frank and G.M. Muller et al., 2018. Fiber-Optic current sensor tolerant to imperfections of polarization-maintaining fiber connectors. J. Lightwave Technol., 36: 2161-2165.

Ciarnoscki, P.F., K. Hoffmann and R.G. Scortegagna, 2015. Improvement of a mobile autonomous robot to participate in sumo competitions. Proceedings of the 2015 IEEE 24th International Symposium on Industrial Electronics (ISIE), June 3-5, 2015, IEEE, Buzios, Brazil, ISBN:978-1-4673-7554-2, pp: 633-637.

Erdem, H., 2011. Application of neuro-fuzzy controller for sumo robot control. Expert Syst. Appl., 38: 9752-9760.

Gacharna, T.A.N., A.D.A. Rodriguez and C. Barbosa, 2016. Learning strategies in mobile and industrial robotics for people with auditory impairment. Proceedings of the 2016 International Conference on Global Humanitarian Technology (GHTC), October 13-16, 2016, IEEE, Seattle, Washington, ISBN:978-1-5090-2432-2, pp: 836-841.

Lima, C.E.O., F.M.A. De Araujo, M.B. Da Silva, R.A.E. Filho and R.D.A.L. Rabelo et al., 2013. Velocity and direction planning in a sumo robot type using the method of potential field with fuzzy systems. Proceedings of the 2013 16th International Conference on Advanced Robotics (ICAR), November 25-29, 2013, IEEE, Montevideo, Uruguay, ISBN:978-1-4799-2722-7, pp: 1-7.

Orefice, P.H., M. Ammi, M. Hafez and A. Tapus, 2016. Let's handshake and I'1l know who you are: Gender and personality discrimination in human-human and human-robot handshaking interaction. Proceedings of the 2016 IEEE-RAS 16th International Conference on Humanoid Robots (Humanoids), November 15-17, 2016, IEEE, Cancun, Mexico, ISBN:978-1-5090-4718-5, pp: 958-965.

Qidwai, U., M. Shakir and O.B. Connor, 2013. Robotic toys for autistic children: Innovative tools for teaching and treatment. Proceedings of the 2013 7th IEEE International Conference on GCC and Exhibition (GCC), November 17-20, 2013, IEEE, Doha, Qatar, ISBN:978-1-4799-0722-9, pp: 188-192.

Siregar, R.H.S., 2017. The implantation of the wireless communication $433 \mathrm{MHz}$ use APC220 a transceiver on the robot music Angklung. Teknosi, 3: 139-139.

$\mathrm{Su}, \mathrm{X}$, Q. Xu and H. Chen, 2017. A new optical voltage sensor for linear measurement. Proceedings of the 2017 2nd IEEE International Conference on Integrated Circuits and Microsystems (ICICM), November 8-11, 2017, IEEE, Nanjing, China, ISBN:978-1-5386-3505-6, pp: $147-150$. 
Victor, H., T. Costachioiu and R. Constantinescu, 2013. Building your own sumo robot in some small simple steps. Proceedings of the 2013 International Conference on Electronics, Computers and Artificial Intelligence (ECAI), June 27-29, 2013, IEEE, Pitesti, Romania, ISBN:978-1-4673-4935-2, pp: 1-4.
Yamato, Y., M. Jindai and T. Watanabe, 2008. Development of a shake-motion leading model for human-robot handshaking. Proceedings of the 2008 International Annual Conference on SICE, August 20-22, 2008, IEEE, Tokyo, Japan, ISBN: 978-4-907764-30-2, pp: 502-507. 